\title{
Packet Acquisition Evaluation of Slotted Spread ALOHA Data Networks
}

\author{
Waseem Jibrail and Ranjith Liyana-Pathirana \\ University of Western Sydney \\ School of Engineering and Industrial Design \\ Locked Bag 1797, Penrith South DC 1797, NSW, Australia
}

Email:w.jibrail@uws.edu.au_r.liyanapathirana@uws.edu.au

\begin{abstract}
In this paper packet acquisition performances of both slotted spread ALOHA and spread ALOHA multiple-access schemes are examined and compared. The probability of packet acquisition of both schemes are measured as users compete to gain access to the network hub receiver in the presence of additive white Gaussian noise (AWGN) and narrowband interference. Moreover, channel load sensing protocol (CLSP) is employed at the receiver hub in order to improve packet acquisition performance.

Results clearly show that the slotted spread ALOHA scheme outperforms spread ALOHA in terms of the probability of packet acquisition. It is also shown that this probability may be improved further by employing the CLSP at the receiver hub.
\end{abstract}

Key words: Spread Spectrum Synchronisation, Spread Aloha

\section{INTRODUCTION}

A spread ALOHA hub station in a data network need only be capable of synchronising to the received signals, all of which use the same Pesudonoise (PN) code, a much simpler problem than that faced by CDMA hub, where PN-codes received all different. On the other hand, the problem of packet acquisition (also known as PN-code acquisition) imposes a limitation on the network capacity, as the capacity of wireless data-networks is essentially limited by the number of simultaneous users that can achieve and maintain The original version of this chapter was revised: The copyright line was incorrect. This has been
corrected. The Erratum to this chapter is available at DOI: $10.1007 / 978-0-387-35618-1 \_37$ 
packet acquisition, rather than by the number of users that can maintain a certain bit error-rate during the data-demodulation process [1]. Using only one pseudonoise (PN) code, spread ALOHA multiple-access scheme users compete to gain access of the receiver hub at any time, while in slottedspread ALOHA users are only allowed to transmit their packets in the beginning of each time slot as shown in Fig.1. Recently, packet acquisition performance (in terms of the probability of packet acquisition $\mathrm{P}_{\mathrm{a}}$ ) of a spread ALOHA scheme for wireless data-networks has been evaluated in the presence of AWGN and narrow band interference [2]. Each user in the network is initially required to gain access to the receiver hub by transmitting a preamble (i.e. a packet without data).

The network hub employs one simple serial-search receiver based on a non-coherent digital sliding correlator $[3,4]$. The serial-search receiver operates by receiving the direct sequence (DS) signal, then searching serially for the correct signal phase using a detector, namely, a digital digital sliding correlator. It was shown [2] that the probability of successful packet acquisition depends on the operating characteristics of the receiver (i.e. probability of detection $P_{d}$ and probability of false alarm $P_{f}$ ), the number of users $r$ and the code length and it is expressed as:

$$
P_{a}=\frac{2 P_{d}}{\left(2-P_{d}\right)\left(1+r . P_{f}\right) \cdot 2 r\left(2^{n}-1\right)} \ldots \ldots \ldots .(1)
$$

For a given receiver parameters such as, bandwidth $\mathrm{B}$, signal-to-noise ratio (SNR), threshold level $V_{n t h}$ and dwell-time (i.e. integration time) $T_{d}, P_{d}$ and $P_{f}$ may be expressed as [5].

$$
\begin{aligned}
& p_{f}=Q\left[\left(V_{n t h}-1\right) \sqrt{B T_{d}}\right] \ldots \ldots \ldots \ldots \ldots \ldots \ldots \ldots \ldots \ldots \ldots \\
& \left.P_{d}=Q \mid\left(V_{n t h}-1-S N R\right) \sqrt{B T_{d} /(1+2 . S N R)}\right]
\end{aligned}
$$

Where

$$
Q(x)=\frac{1}{2 \pi} \int_{0}^{\infty} \exp \left(-u^{2} / 2\right) d u
$$

Furthermore, the type of receiver employed by the hub and its key parameters have either a direct or indirect effect on packet acquisition 
performance and hence the probability of packet acquisition. Moreover, it was shown that [2-4] adaptive threshold detection at the receiver hub plays a significant role in improving the probability of packet acquisition.

To further improve the packet acquisition performance of spread ALOHA, a channel load sensing protocol (CLSP) $[6,7]$ may be employed at the hub station receiver. In CLSP the hub senses the channel load status (i.e. number of packets being transmitted). If channel load is over a certain

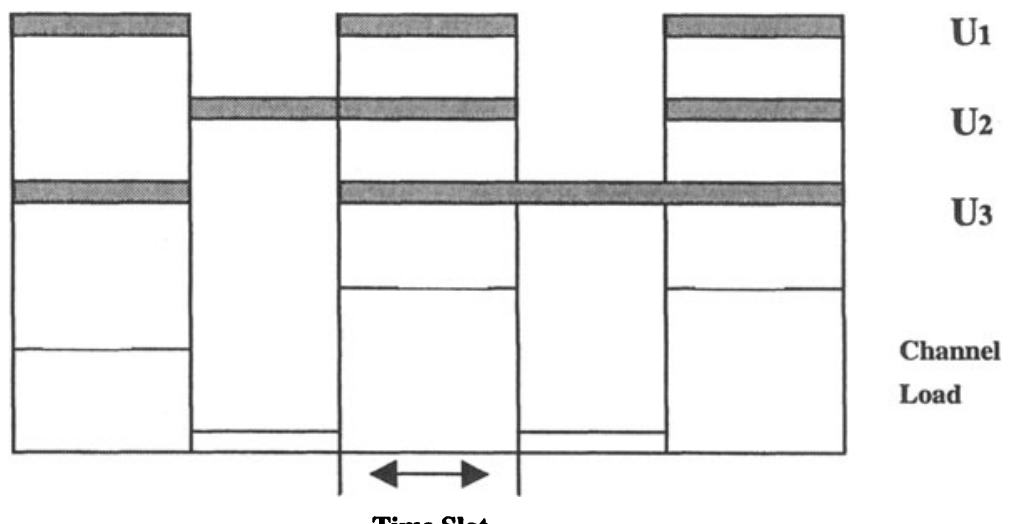

Time Slot

Fig.1 Slotted Spread ALOHA

Channel

threshold, then packet access to the hub is rejected until the channel load falls below threshold. In this way a maximum number of users are allowed to access the network hub for synchronisation purposes.

\section{PERFORMANCE COMPARASION}

The up-link part of the network, shown in Fig.2, is implemented using MATLAB. As network users transmit their packets simultaneously, they are also kept at different distances from the hub receiver by randomly varying the initial phase of the transmitted PN-codes in each run. Variable initial phase is realised by randomly delaying the outputs of all transmitters as shown in Fig.2. Packets transmission are only allowed at the beginning of each time slot using gating. Extensive simulations are conducted of the slotted Spread ALOHA scheme to measure the probability of packet acquisition in the presence of AWGN, narrowband interference and multiple-access-interference (MAI). Fig.3. shows the probability of packet acquisition as a function of correlation length of the digital sliding correlator 


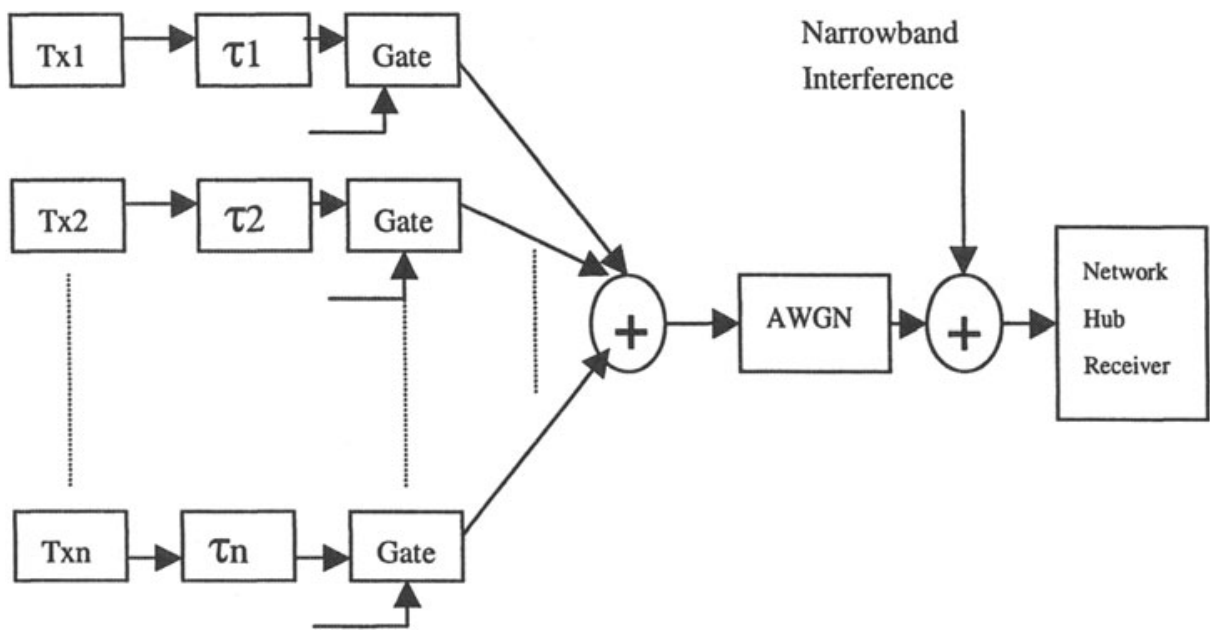

Fig.2 Block Diagram of Simulated Network

for both slotted spread ALOHA and spread ALOHA. Results were obtained for a signal-to-noise ratio (SNR) of $0 \mathrm{~dB}$, interference-to-signal ratio (ISR ) of $12.5 \mathrm{~dB}$ and $\mathrm{PN}$-code length of $\mathrm{L}=255$ chips.

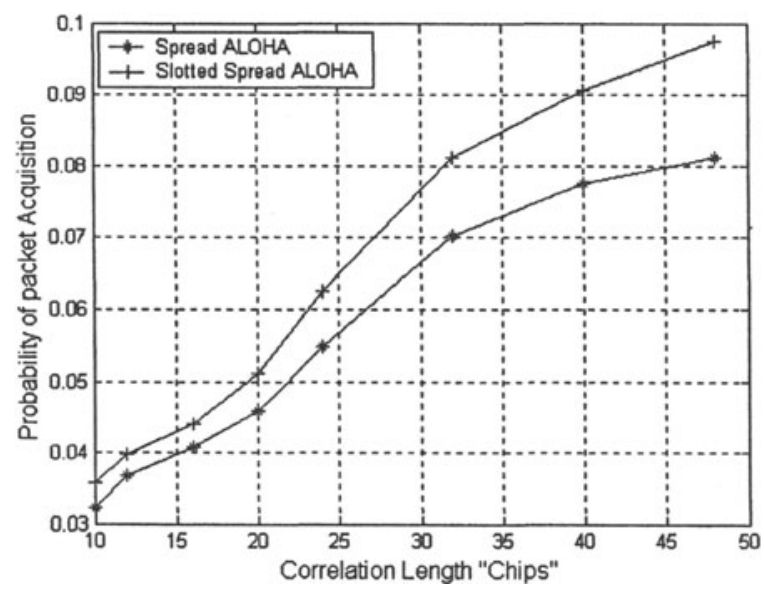

Fig.3 $\mathrm{P}_{\mathrm{a}}$ as a function of correlation length

Fig.4 shows $\mathrm{P}_{\mathrm{a}}$ as a function of ISR for adaptive and constant threshold detection at $\mathrm{SNR}=0 \mathrm{~dB}$, for both spread and slotted spread ALOHA schemes. while, Fig.5 illustrates $P_{a}$ as a function of number of users for both spread and slotted spread ALOHA schemes and at $\mathrm{SNR}=0 \mathrm{~dB}$ and ISR $=$ $12.5 \mathrm{~dB}$. Clearly, simulation results demonstrate that the performance of the slotted spread ALOHA scheme is superior to the spread ALOHA scheme in 


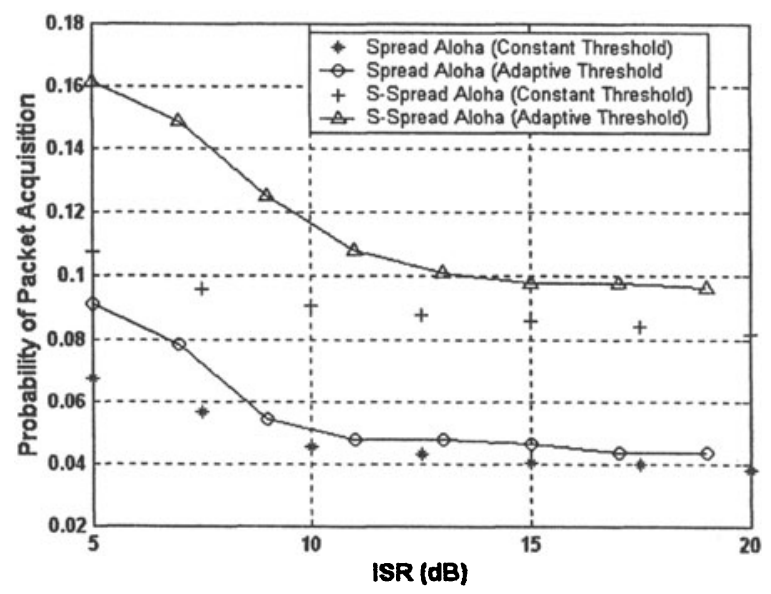

Fig.4 $P_{a}$ as a function of ISR

terms of the probability of acquisition for the same receiver at the network hub. This gain in the probability of packet acquisition is significant when adaptive threshold detection is employed at the receiver. Table I illustrates this improvement in $\mathrm{P}_{\mathrm{a}}$ for different ISR 's and different number of users $r$.

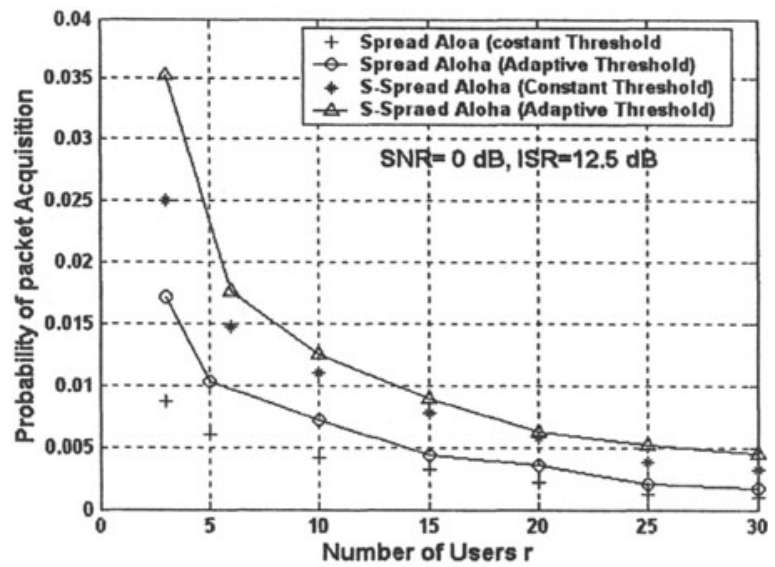

Fig.5 $\mathrm{P}_{\mathrm{a}}$ as a function of number of users

Generally, results indicate that as MAI (number of users) and narrowband interference (ISR) both increase, the gain in $\mathrm{P}_{\mathrm{a}}$ also increases. This stems from the fact that adaptive threshold detection, employed by the receiver at the network hub, enjoys better performance, in contrast to constant 
threshold, particularly in high interference environments [4]. In this way network capacity may be enhanced.

\begin{tabular}{|c|c|c|c|}
\hline $\begin{array}{c}r \\
\text { users }\end{array}$ & $\begin{array}{c}\text { Gain } \\
\text { in } \mathrm{P}_{\mathrm{a}}\end{array}$ & $\begin{array}{c}\text { IS } \\
\text { R dB }\end{array}$ & $\begin{array}{c}\text { Gain } \\
\text { in } \mathrm{P}_{\mathrm{a}}\end{array}$ \\
\hline 3 & 2 & 5 & 1.7 \\
\hline 5 & 1.7 & 7 & 1.9 \\
\hline 10 & 1.7 & 9 & 2.31 \\
\hline 15 & 2 & 11 & 2.14 \\
\hline 20 & 2 & 13 & 2.1 \\
\hline 25 & 2.5 & 15 & 2.13 \\
\hline 30 & 2.6 & 17 & 2.25 \\
\hline
\end{tabular}

Table I Gain in $\mathrm{P}_{\mathrm{a}}$ of Slotted Spread

ALOHA over Spread ALOHA using Adaptive

Threshold

\section{SLOTTED SPREAD ALOHA WITH CLSP}

To further improve the performance of slotted spread ALOHA scheme, in terms of the probability of packet acquisition, channel load sensing protocol (CLSP) $[6,7]$ is employed to control the maximum number of users competing to gain access of the network receiver hub station in a given cell at one time. This is performed by monitoring the channel load and applying a threshold to limit the number of users that are allowed to access the channel at any one time. Two threshold levels are examined ,these are; $(2 / 3)$ $r$ and $(1 / 3) r$, where $r$ is the total number of users competing to gain access to the same receiver, for acquisition purposes, at the network receiver hub.

Fig.6 illustrates the probability of packet acquisition as a function of ISR with and without CLSP using adaptive threshold at the hub receiver. This indicates clearly that slotted ALOHA scheme may perform much better with CLSP (i.e. as the number of users are controlled during initial synchronisation). 


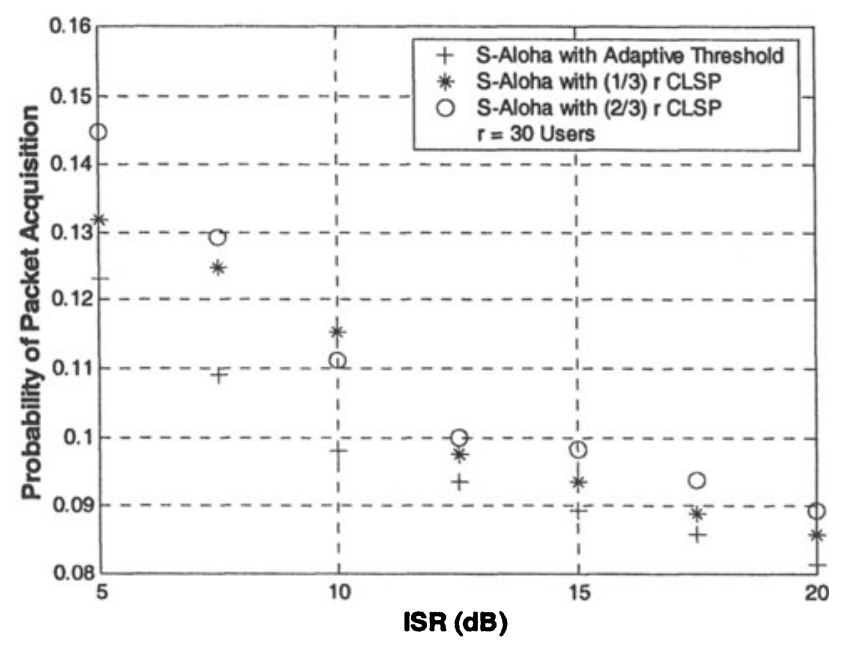

Fig.6 Probability of Packet Acquisition against ISR with CLSP

\section{CONCLUSION}

The probability of packet acquisition for both slotted spread ALOHA and spread ALOHA schemes are compared in the presence of AWGN, narrrowband interference and MAI. Results signify the superior performance of slotted ALOHA scheme for the same serial-search receiver employed at the network hub. Thus, network capacity may be improved.

It is shown that incorporating channel load control protocol (CLSP) with slotted spread ALOHA further enhances its performance in terms of the probability of packet acquisition.

\section{REFERENCES}

[1] Madhow U and Pursley M.B,"Acquisition in Direct-Sequence Spread-Spectrum

Communication Networks: An Asymptotic Analysis", IEEE Trans. On Information Theory,Vol39, No.3, May 1993, pp. 903-912.

[2] Jibrail W and Liyana-Pathirana R "Packet Acquisition Performance of Spread ALOHA Wireless Data Networks", Proc. Of the third International Conference on Information, Communications and Signal Processing (ICICS 2001), ISBN 981-04-5149-0, P0125, Oct. 15-18,2001, Singapore. 
[3] Jibrail W, Liyana-Pathirana R, Robert FRY," A Modified Serial-Search DS CodeAcquisition Scheme", IEEE Sixth International Symposium on Signal Processing and its Applications (ISSPA 2001),13-16 August, Catalog No. 01EX467C, ISBN 0-7803-6704-9, 2001 IEEE, Kuala Lampur, Malaysia.

[4] Jibrail W,"DS Spread Spectrum Signal Acquisition Using Adaptive Threshold Techniques", Int. Jour. Commun. Syst., Vol. 11, 1998, pp. 297-303.

[5] Simon,M.K., Omura, J.K, Scholtz R.A and Levitt B.K,"Spread Spectrum Communications, Vol. III, Rockville,MD: computer press 1985.

[6] K. Tohimitsu, T.Yamazato, M. Katayama and A. Ogawa,"A Novel Spread Slotted ALOHA System with Channel Load Sensing Protocol", IEEE Jour. On Selected Areas in Communications (JSAC), Vol.12, No. 4, May 1994, pp. 655-672.

[7] H. Okada, M. Saito, T.Yamazato, M. Katayama and A. Ogawa, "Performance Evaluation of CDMA ALOHA Systems with Channel Load Sensing Protocol", Global Communications Conference, Vol. 2, Nov. 12-18, 1996, pp. 1291-1295. 